\title{
Robust Control of Large Vehicular Platoons with Prescribed Transient and Steady State Performance
}

\author{
Charalampos P. Bechlioulis, Dimos V. Dimarogonas and Kostas J. Kyriakopoulos
}

\begin{abstract}
In this paper, we consider the longitudinal control problem for a platoon of vehicles with unknown nonlinear dynamics. More specifically, we design a decentralized modelfree control protocol in the sense that each vehicle utilizes only local relative information regarding its front vehicle, obtained by its on-board sensors, to calculate its own control signal, without incorporating any prior knowledge of the model nonlinearities/disturbances or any approximation structures to acquire such knowledge. Additionally, the transient and steady state response is a priori determined by certain designerspecified performance functions and is fully decoupled by the agents' dynamic model, the number of vehicles composing the platoon and the control gains selection, which relaxes significantly the control design procedure. Moreover, introducing certain inter-vehicular distance constraints during the transient response shaping, collisions between successive vehicles as well as connectivity breaks owing to limited sensing capabilities are provably avoided. Finally, the proposed methodology results in a low complexity design. Actually, it is a static scheme involving very few and simple calculations to output the control signal, thus making its distributed implementation straightforward.
\end{abstract}

\section{INTRODUCTION}

Automated highway systems have become an active research area in automatic control since early 90's, in an attempt to improve the capacity (measured in vehicles/lanes/time) and safety of highways via controlling the inter-vehicular distance. Early works on the longitudinal control for large platoons of vehicles employed optimal centralized control to regulate the string of moving vehicles [1]-[4]. However, feedback from all vehicles had to be continually transmitted to a central controller or to all vehicles, thus necessitating for high communication overhead and making it less attractive for platoons with a large number of vehicles, since the closed loop system becomes inherently sensitive to delays, inevitable in wireless communication.

In such case, a decentralized architecture, where the control action of each vehicle is computed based upon measurements from its on-board sensors, is definitely much more efficient. Towards this direction, two decentralized control architectures have been developed in the related literature, namely the predecessor-following [5]-[7] and the bidirectional schemes [7]-[11]. More specifically, in the predecessor-following architecture, the control action of each

C. P. Bechlioulis and K. J. Kyriakopoulos are with the Control Systems Laboratory, School of Mechanical Engineering, National Technical University of Athens, Athens 15780, Greece. D. V. Dimarogonas is with the Centre for Autonomous Systems at Kungliga Tekniska Hogskolan, Stockholm 10044, Sweeden. Emails: chmpechl@mail.ntua.gr, dimos@kth.se, kkyria@mail.ntua.gr.

This work was supported by the EU funded project RECONFIG: Cognitive, Decentralized Coordination of Heterogeneous Multi-Robot Systems via Reconfigurable Task Planning, FP7-ICT-600825, 2013-2016. vehicle depends only on the relative information from its immediate predecessor (i.e., the vehicle in front of it), whereas in the bidirectional architecture, the control depends upon relative measurements from both the predecessor and the follower.

Although the majority of the works on the aforementioned decentralized architectures consider known (or partially known) and simple dynamic models, in practice, however, the vehicle dynamics cannot be modeled accurately and are constantly subject to unknown environmental disturbances (e.g., wind gust). Thus, taking into account the inherent model uncertainties, when designing decentralized control schemes, is of paramount importance as they may cause slinky type effects, and therefore, may result in the loss of platoon stability. On the other hand, extending towards this direction the existing control schemes, becomes a very challenging task on account of the increasing design complexity from the interacting system dynamics as reflected by the local intercourse specifications. Another important issue associated with decentralized control schemes for large platoons of vehicles, concerns the transient and steady state response of the closed loop system. Traditionally, model uncertainties affect seriously the performance of the controlled vehicle, whereas the sensitivity of the closed loop system to external disturbances increases as the number of vehicles increases. More specifically, the error is proven to convergence within a residual set, whose size depends on control design parameters and some unknown (though bounded) terms induced by the model uncertainties and the external disturbances. However, no systematic procedure exists to accurately compute the required upper bounds, thus making the a priori selection of the aforementioned control parameters to satisfy certain steady state behavior, practically impossible. Additionally, the transient behavior is difficult to establish analytically, even in case of known models, as it is heavily affected by the number of the vehicles comprising the platoon and the model parameters.

In this work, we propose a decentralized control protocol for large platoons of vehicles with uncertain nonlinear dynamics, based on the predecessor-following architecture, that creates arbitrarily fast and maintains with arbitrary accuracy a desired feasible formation without any intervehicular collisions and connectivity breaks (owing to limited sensing capabilities). The developed scheme exhibits the following important characteristics. First, it is purely decentralized in the sense that the control signal of each agent is calculated based solely on local relative information from its on-board sensors. Furthermore, its complexity proves to 
be considerably low. Very few and simple calculations are required to output the control signal. Additionally, it does not require any prior knowledge of the vehicle's dynamic model and no estimation models are employed to acquire such knowledge. Moreover, contrary to the related works, the transient and steady state response is fully decoupled by the number of vehicles composing the platoon, the control gains selection and the vehicles' model uncertainties. In particular, the achieved performance as well as the collision avoidance and the connectivity maintenance are a priori and explicitly imposed by certain designer-specified performance functions, thus simplifying significantly the selection of the control gains. Tuning of the controller gains is only confined to achieving reasonable control effort.

\section{Problem Statement and Preliminaries}

We consider the formation control problem of $N$ vehicles moving in 1-D Euclidean space with 2nd order nonlinear dynamics:

$$
\begin{aligned}
\dot{p}_{i} & =v_{i} \\
m_{i} \dot{v}_{i} & =f_{i}\left(v_{i}\right)+u_{i}+w_{i}(t), i=1, \ldots, N
\end{aligned}
$$

where $p_{i}$ and $v_{i}$ denote the position and velocity of each vehicle respectively, $m_{i}$ is the mass, which is considered unknown, $f_{i}\left(v_{i}\right)$ is an unknown continuous nonlinear function, $u_{i}$ is the control input and $w_{i}(t)$ is a bounded piecewise continuous function of time representing exogenous disturbances. The control objective is to design a distributed control protocol such that a rigid formation is established with prescribed transient and steady state performance, despite the presence of model uncertainties. By prescribed performance, we mean that the formation is achieved in a predefined transient period and is maintained arbitrarily accurate while avoiding collisions and connectivity breaks with neighboring vehicles. The geometry of the formation is represented by the desired gaps $\Delta_{i-1, i}, i=1, \ldots, N$ between two consecutive vehicles (see Fig. 1), where $\Delta_{i-1, i}>$ 0 denotes the desired distance between the $(i-1)$-th and $i$ th vehicle (i.e., $\left.p_{i}(t) \rightarrow p_{i-1}(t)-\Delta_{i-1, i}\right)$. Moreover, the distance $p_{i-1}(t)-p_{i}(t)$ should be kept greater than $\Delta_{\text {col }}$ to avoid collisions and less than $\Delta_{\text {con }}$ to maintain the network connectivity owing to the limited sensing capabilities of the vehicles (e.g., when employing range finders to measure the distance between two succussive vehicles). Furthermore, to ensure the feasibility of the desired formation we assume that $\Delta_{\text {col }}<\Delta_{i-1, i}<\Delta_{\text {con }}, i=1, \ldots, N$. Additionally, the desired trajectory of the formation is generated by a reference/leading vehicle denoted by $p_{0}(t)$ with bounded velocity $v_{0}(t)$ and is only provided to the first vehicle. Finally, to solve the aforementioned formation control problem, the following assumption is required.

Assumption A1. The initial state of the platoon does not violate the collision and connectivity constraints. That is $\Delta_{\text {col }}<p_{i-1}(0)-p_{i}(0)<\Delta_{\text {con }}, i=1, \ldots, N$.

In this work, we consider the predecessor-following architecture, according to which the control action of each vehicle

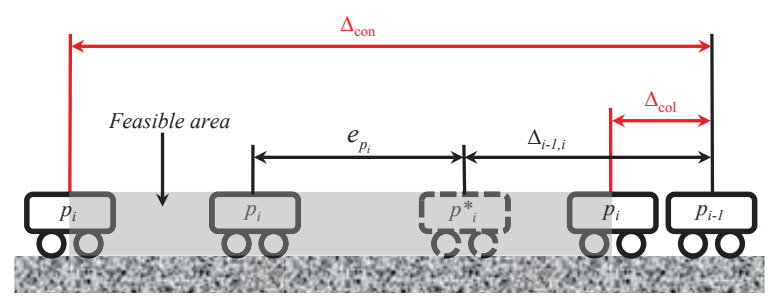

Fig. 1. Graphical illustration of two consecutive vehicles of the platoon. The desired position of the $i$-th vehicle with respect to its predecessor is denoted by $p_{i}^{\star}=p_{i-1}-\Delta_{i-1, i}$. Furthermore, each vehicle should keep its distance to the preceding one within the feasible area $\Delta_{\text {col }}<p_{i-1}(t)-$ $p_{i}(t)<\Delta_{\text {con }}$, thus avoiding collisions and connectivity breaks.

is based only on its preceding vehicle. Thus, we formulate $N$ control variables as follows:

$$
e_{p_{i}}(t)=p_{i-1}(t)-p_{i}(t)-\Delta_{i-1, i}, i=1, \ldots, N .
$$

Since the aforementioned problem is represented by a strongly connected directed graph, we may also define the neighborhood error vector $e_{p}=\left[e_{p_{1}}, \ldots, e_{p_{N}}\right]^{T}$ with respect to the leading vehicle as follows:

$$
e_{p}=-\tilde{L}\left(p-\bar{p}_{0}+\bar{\Delta}_{0}\right)
$$

where $p=\left[p_{1}, \ldots, p_{N}\right]^{T}, \bar{p}_{0}:=\left[p_{0}, \ldots, p_{0}\right]^{T}, \bar{\Delta}_{0}:=$ $\left[\Delta_{0,1}, \Delta_{0,2}, \ldots, \Delta_{0, N}\right]^{T}$ with $\Delta_{0, i}=\sum_{j=1}^{i} \Delta_{j-1, j}, i=$ $1, \ldots, N$ and $\tilde{L}$ is the lower bidiagonal augmented Laplacian [12] of the graph, which has strictly positive singular values owing to the strong connectivity of the considered directed graph [13]. Moreover, since all principal minors of $\tilde{L}$ equal to 1 then $\tilde{L}$ is also a nonsingular $\mathcal{M}$-matrix [14]. Finally, the following technical lemma plays an important role in the subsequent analysis.

Lemma 1: [13] For a nonsingular $\mathcal{M}$-matrix $A \in$ $\Re^{N \times N}$, there exists a diagonal positive definite matrix $P=$ $\left(\operatorname{diag}\left(A^{-1} \underline{1}\right)\right)^{-1}$ such that the matrix $Q=P A+A^{T} P$ is positive definite as well with $\underline{1}$ denoting the $N$-th dimensional vector of ones.

\section{A. Prescribed Performance}

The control scheme is connected to the prescribed performance notion that was originally proposed to design robust state feedback controllers, for various classes of nonlinear systems [15]-[17], capable of guaranteeing output tracking with prescribed performance. In this work, by prescribed performance, it is meant that the output tracking error converges to a predefined arbitrarily small residual set with convergence rate no less than a certain predefined value. For completeness and compactness of presentation, this chapter summarizes preliminary knowledge on prescribed performance. In that respect, consider a generic scalar tracking error $e(t)$. Prescribed performance is achieved if $e(t)$ evolves strictly within a predefined region that is bounded by certain functions of time. The mathematical expression of prescribed performance is given by the following inequalities:

$$
-\rho_{L}(t)<e(t)<\rho_{U}(t), \forall t \geq 0
$$


where $\rho_{L}(t), \rho_{U}(t)$ are smooth and bounded functions of time satisfying $\lim _{t \rightarrow \infty} \rho_{U}(t)>0$ and $\lim _{t \rightarrow \infty} \rho_{L}(t)>0$, called performance functions. Specifically, for the exponential performance functions $\rho_{i}(t)=\left(\rho_{i 0}-\rho_{i \infty}\right) e^{-l_{i} t}+\rho_{i \infty}$ with $\rho_{i 0}, \rho_{i \infty}, l_{i}, i \in\{L, U\}$ appropriately chosen constants, the constants $\rho_{L 0}=\rho_{L}(0), \rho_{U 0}=\rho_{U}(0)$ are selected such that $\rho_{U 0}>e(0)>-\rho_{L 0}$ and the constants $\rho_{L \infty}=$ $\lim _{t \rightarrow \infty} \rho_{L}(t), \rho_{U \infty}=\lim _{t \rightarrow \infty} \rho_{U}(t)$ represent the maximum allowable size of the tracking error $e(t)$ at the steady state, which may even be set arbitrarily small to a value reflecting the resolution of the measurement device, thus achieving practical convergence of $e(t)$ to zero. Moreover, the decreasing rate of $\rho_{L}(t), \rho_{U}(t)$ which is affected by the constants $l_{L}, l_{U}$ in this case, introduces a lower bound on the required speed of convergence of $e(t)$. Therefore, the appropriate selection of the performance functions $\rho_{L}(t)$, $\rho_{U}(t)$ imposes performance characteristics on the tracking error $e(t)$.

\section{B. Dynamical Systems}

Consider the initial value problem:

$$
\dot{\psi}=H(t, \psi), \psi(0)=\psi^{0} \in \Omega_{\psi}
$$

with $H: \Re_{+} \times \Omega_{\psi} \rightarrow \Re^{n}$ where $\Omega_{\psi} \subset \Re^{n}$ is a non-empty open set.

Definition 1: [18] A solution $\psi(t)$ of the initial value problem (4) is maximal if it has no proper right extension that is also a solution of (4).

Theorem 1: [18] Consider the initial value problem (4). Assume that $H(t, \psi)$ is: a) locally Lipschitz on $\psi$ for almost all $t \in \Re_{+}$, b) piecewise continuous on $t$ for each fixed $\psi \in \Omega_{\psi}$ and c) locally integrable on $t$ for each fixed $\psi \in \Omega_{\psi}$. Then, there exists a maximal solution $\psi(t)$ of (4) on the time interval $\left[0, t_{\max }\right)$ with $t_{\max }>0$ such that $\psi(t) \in \Omega_{\psi}$, $\forall t \in\left[0, t_{\max }\right)$.

Proposition 1: [18] Assume that the hypotheses of Theorem 1 hold. For a maximal solution $\psi(t)$ on the time interval $\left[0, t_{\max }\right)$ with $t_{\max }<\infty$ and for any compact set $\Omega_{\psi}^{\prime}$ $\subset \Omega_{\psi}$ there exists a time instant $t^{\prime} \in\left[0, t_{\max }\right)$ such that $\psi\left(t^{\prime}\right) \notin \Omega_{\psi}^{\prime}$.

\section{CONTROL DESIGN}

In this work, prescribed performance control will be adopted in order: i) to achieve predefined transient and steady state response for each neighborhood position error $e_{p_{i}}(t)$, $i=1, \ldots, N$ as well as ii) to avoid the violation of the collision and connectivity constraints as presented in Section II. Following Subsection II-A, prescribed performance is achieved when the neighborhood position errors $e_{p_{i}}(t), i=$ $1, \ldots, N$ evolve strictly within a predefined region that is bounded by absolutely decaying functions of time, called performance functions. The mathematical expression of prescribed performance is given by the following inequalities:

$$
-\underline{M}_{p_{i}} \rho_{p_{i}}(t)<e_{p_{i}}(t)<\bar{M}_{p_{i}} \rho_{p_{i}}(t), \forall t \geq 0
$$

for all $i=1, \ldots, N$, where:

$$
\rho_{p_{i}}(t)=\left(1-\frac{\rho_{\infty}}{\max \left\{\underline{M}_{p_{i}}, \bar{M}_{p_{i}}\right\}}\right) e^{-l t}+\frac{\rho_{\infty}}{\max \left\{\underline{M}_{p_{i}}, \bar{M}_{p_{i}}\right\}}
$$

are designer-specified, smooth, bounded and decreasing functions of time with $l, \rho_{\infty}$ positive parameters incorporating the desired transient and steady state performance specifications respectively, and $\underline{M}_{p_{i}}, \bar{M}_{p_{i}}, i=1, \ldots, N$ positive parameters selected appropriately to satisfy the collision and connectivity constraints, as presented in the sequel. In particular, the decreasing rate of $\rho_{p_{i}}(t), i=1, \ldots, N$ which is affected by the constant $l$, introduces a lower bound on the speed of convergence of $e_{p_{i}}(t), i=1, \ldots, N$. Furthermore, the constant $\rho_{\infty}$ can be set arbitrarily small, thus achieving practical convergence of $e_{p_{i}}(t), i=1, \ldots, N$ to zero. Additionally, we select:

$$
\left.\begin{array}{c}
\underline{M}_{M_{i}}=\Delta_{i-1, i}-\Delta_{\text {col }} \\
p_{i} \\
=\Delta_{\text {con }}-\Delta_{i-1, i}
\end{array}\right\}, i=1, \ldots, N .
$$

Apparently, since the desired formation is compatible with the collision and connectivity constraints (i.e., $\Delta_{\text {col }}<$ $\left.\Delta_{i-1, i}<\Delta_{\text {con }}, i=1, \ldots, N\right)$, the aforementioned selection ensures that $\underline{M}_{p_{i}}, \bar{M}_{p_{i}}>0, i=1, \ldots, N$ and consequently under Assumption $\mathbf{A} 1$ (i.e., $\Delta_{\text {col }}<p_{i-1}(0)-p_{i}(0)<\Delta_{\text {con }}$, $i=1, \ldots, N)$ that:

$$
-\underline{M}_{p_{i}} \rho_{p_{i}}(0)<e_{p_{i}}(0)<\bar{M}_{p_{i}} \rho_{p_{i}}(0), i=1, \ldots, N .
$$

Hence, guaranteeing prescribed performance via (5) and employing the decreasing property of $\rho_{p_{i}}(t), i=1, \ldots, N$, we obtain:

$$
-\underline{M}_{p_{i}}<e_{p_{i}}(t)<\bar{M}_{p_{i}}, \forall t \geq 0
$$

and consequently, owing to (7):

$$
\Delta_{\text {col }}<p_{i-1}(t)-p_{i}(t)<\Delta_{\text {con }}, \forall t \geq 0
$$

which ensures that the collision and connectivity constraints are satisfied for all $t \geq 0$.

In the sequel, we propose a distributed control protocol that does not incorporate any information on the vehicles' nonlinear model or the external disturbances and guarantees $-\underline{M}_{p_{i}} \rho_{p_{i}}(t)<e_{p_{i}}(t)<\bar{M}_{p_{i}} \rho_{p_{i}}(t), i=1, \ldots, N$ for all $t \geq 0$, thus leading to the solution of the robust formation control problem with prescribed performance under collision and connectivity constraints for the considered platoon of vehicles.

\section{A. Decentralized Predecessor-Following Control Protocol}

The distributed control design is twofold. Given the neighborhood position errors $e_{p_{i}}(t)=p_{i-1}(t)-p_{i}(t)-\Delta_{i-1, i}$, $i=1, \ldots, N$ :

Step I-a. Select the corresponding functions $\rho_{p_{i}}(t)$ and positive parameters $\underline{M}_{p_{i}}, \bar{M}_{p_{i}}, i=1, \ldots, N$ following (6) and (7) respectively, in order to incorporate the desired transient and steady state performance specifications as well as the collision and connectivity constraints.

Step I-b. Define the normalized neighborhood position errors as:

$$
\xi_{p}\left(e_{p}, t\right)=\left[\begin{array}{c}
\xi_{p_{1}}\left(e_{p_{1}}, t\right) \\
\vdots \\
\xi_{p_{N}}\left(e_{p_{N}}, t\right)
\end{array}\right] \triangleq\left(\rho_{p}(t)\right)^{-1} e_{p}
$$


where $\rho_{p}(t)=\operatorname{diag}\left(\left[\rho_{p_{i}}(t)\right]_{i=1, \ldots, N}\right)$ and design the decentralized reference velocity vector as:

$$
\begin{aligned}
v_{d}\left(\xi_{p}, t\right) & =\left[\begin{array}{c}
v_{d_{1}}\left(\xi_{p_{1}}, t\right) \\
\vdots \\
v_{d_{N}}\left(\xi_{p_{N}}, t\right)
\end{array}\right] \\
& =k_{p}\left(\rho_{p}(t)\right)^{-1} r_{p}\left(\xi_{p}\right) \varepsilon_{p}\left(\xi_{p}\right)
\end{aligned}
$$

with $k_{p}>0$ and

$$
\begin{aligned}
& r_{p}\left(\xi_{p}\right)=\operatorname{diag}\left(\left[\frac{\frac{1}{\underline{M} p_{i}}+\frac{1}{\bar{M}_{p_{i}}}}{\left(1+\frac{\xi_{p_{i}}}{\underline{\underline{M}} p_{i}}\right)\left(1-\frac{\xi_{p_{i}}}{\bar{M}_{p_{i}}}\right)}\right]_{k=1, \ldots, N}\right) \\
& \varepsilon_{p}\left(\xi_{p}\right)=\left[\ln \left(\frac{1+\frac{\xi_{p_{1}}}{\underline{\underline{M}} p_{1}}}{1-\frac{\xi_{p_{1}}}{\bar{M}_{p_{1}}}}\right), \ldots, \ln \left(\frac{1+\frac{\xi_{p_{N}}}{\frac{\underline{M}_{N}}{p_{N}}}}{1-\frac{\xi_{p_{N}}}{\bar{M}_{p_{N}}}}\right)\right]^{T}
\end{aligned}
$$

Step II-a. Define the velocity error vector $e_{v}=$ $\left[e_{v_{1}}, \ldots, e_{v_{N}}\right]^{T}$ with $e_{v_{i}}=v_{i}-v_{d_{i}}\left(e_{p_{i}}, t\right), i=1, \ldots, N$ and select the corresponding velocity performance functions $\rho_{v_{i}}(t), i=1, \ldots, N$ such that $\rho_{v_{i}}(0)>\left|e_{v_{i}}(0)\right|, i=$ $1, \ldots, N$.

Step II-b. Similarly to the first step define the normalized velocity errors as:

$$
\xi_{v}\left(e_{v}, t\right)=\left[\begin{array}{c}
\xi_{v_{1}}\left(e_{v_{1}}, t\right) \\
\vdots \\
\xi_{v_{N}}\left(e_{v_{N}}, t\right)
\end{array}\right] \triangleq\left(\rho_{v}(t)\right)^{-1} e_{v}
$$

where $\rho_{v}(t)=\operatorname{diag}\left(\left[\rho_{v_{i}}(t)\right]_{i=1, \ldots, N}\right)$ and finally design the decentralized control protocol:

$$
u\left(\xi_{v}, t\right)=\left[\begin{array}{c}
u_{1}\left(\xi_{v_{1}}, t\right) \\
\vdots \\
u_{N}\left(\xi_{v_{N}}, t\right)
\end{array}\right]=-k_{v} \varepsilon_{v}\left(\xi_{v}\right), k_{v}>0
$$

with

$$
\varepsilon_{v}\left(\xi_{v}\right)=\left[\ln \left(\frac{1+\xi_{v_{1}}}{1-\xi_{v_{1}}}\right), \ldots, \ln \left(\frac{1+\xi_{v_{N}}}{1-\xi_{v_{N}}}\right)\right]^{T} .
$$

Remark 1: The proposed control protocol, is decentralized in the sense that each vehicle utilizes only local relative information to calculate its own control signal. Moreover, it does not incorporate any prior knowledge of the model nonlinearities/disturbances or even of some corresponding upper/lower bounding functions, relaxing thus significantly the key assumptions made in the related literature. Furthermore, the proposed methodology results in a low complexity design. Notice that no hard calculations (neither analytic nor numerical) are required to output the proposed control signal, thus making its distributed implementation straightforward.

\section{B. Stability Analysis}

The main results of this work are summarized in the following theorem where it is proven that the aforementioned decentralized control protocol solves the robust formation control problem with prescribed performance under collision and connectivity constraints for the considered platoon of vehicles.
Theorem 2: Consider a platoon of $N$ vehicles of the form (1), following a leader in 1-D and aiming at establishing a formation described by the desired gaps $\Delta_{i-1, i}, i=$ $1, \ldots, N$ between consecutive vehicles, while satisfying the collision and connectivity constraints represented by $\Delta_{\text {col }}$ and $\Delta_{\text {con }}$ respectively with $\Delta_{\text {col }}<\Delta_{i-1, i}<\Delta_{\text {con }}, i=1, \ldots, N$. Under Assumption A1, the decentralized control protocol (9)-(15) guarantees:

$$
-\underline{M}_{p_{i}} \rho_{p_{i}}(t)<e_{p_{i}}(t)<\bar{M}_{p_{i}} \rho_{p_{i}}(t), \forall t \geq 0
$$

for all $i=1, \ldots, N$, as well as the boundedness of all closed loop signals.

Proof: Differentiating (9) and (13), employing (1), (2) as well as the fact that $v_{i}=v_{d_{i}}+\rho_{v_{i}}(t) \xi_{v_{i}}$ and substituting (10), (14), we arrive at:

$$
\begin{aligned}
\dot{\xi}_{p}= & h_{p}(t, \xi) \\
= & -k_{p}\left(\rho_{p}(t)\right)^{-1} \tilde{L}\left(\rho_{p}(t)\right)^{-1} r_{p}\left(\xi_{p}\right) \varepsilon_{p}\left(\xi_{p}\right) \\
& -\left(\rho_{p}(t)\right)^{-1}\left(\dot{\rho}_{p}(t) \xi_{p}+\tilde{L}\left(\rho_{v}(t) \xi_{v}-\dot{\bar{p}}_{0}(t)\right)\right)(16) \\
\dot{\xi}_{v}= & h_{v}(t, \xi) \\
= & -k_{v}\left(\rho_{v}(t)\right)^{-1} M^{-1} \varepsilon_{v}\left(\xi_{v}\right)-\left(\rho_{v}(t)\right)^{-1}\left(\dot{\rho}_{v}(t) \xi_{v}\right. \\
& \left.-M^{-1}\left(f\left(v_{d}+\rho_{v}(t) \xi_{v}\right)+w(t)\right)+\dot{v}_{d}\right)
\end{aligned}
$$

where

$$
\begin{aligned}
M & =\operatorname{diag}\left(\left[m_{i}\right]_{i=1, \ldots, N}\right) \\
f\left(v_{d}+\rho_{v}(t) \xi_{v}\right) & =\left[\begin{array}{c}
f_{1}\left(v_{d_{1}}+\rho_{v_{1}}(t) \xi_{v_{1}}\right) \\
\vdots \\
f_{N}\left(v_{d_{N}}+\rho_{v_{N}}(t) \xi_{v_{N}}\right)
\end{array}\right]
\end{aligned}
$$

with $m_{i}, f_{i}(\cdot), i=1, \ldots, N$ denoting the unknown mass and nonlinearity of the vehicle model (1) respectively. Thus, the closed loop dynamical system of $\xi(t)=\left[\xi_{p}^{T}(t), \xi_{v}^{T}(t)\right]^{T}$ may be written in compact form as:

$$
\dot{\xi}=h(t, \xi)=\left[\begin{array}{c}
h_{p}(t, \xi) \\
h_{v}(t, \xi)
\end{array}\right] .
$$

Let us also define the open set $\Omega_{\xi}=\Omega_{\xi_{p}} \times \Omega_{\xi_{v}} \subset \Re^{2 N}$ with:

$$
\begin{aligned}
\Omega_{\xi_{p}} & =\left(-\underline{M}_{p_{1}}, \bar{M}_{p_{1}}\right) \times \cdots \times\left(-\underline{M}_{p_{N}}, \bar{M}_{p_{N}}\right) \\
\Omega_{\xi_{v}} & =\underbrace{(-1,1) \times \cdots \times(-1,1)}_{N \text {-times }} .
\end{aligned}
$$

In what follows, we proceed in two phases. First, the existence of a unique maximal solution $\xi(t)$ of (18) over the set $\Omega_{\xi}$ for a time interval $\left[0, \tau_{\max }\right.$ ) (i.e., $\xi(t) \in \Omega_{\xi}, \forall t \in$ $\left.\left[0, \tau_{\max }\right)\right)$ is ensured. Then, we prove that the proposed control scheme (10) and (14) guarantees, for all $t \in\left[0, \tau_{\max }\right)$ : a) the boundedness of all closed loop signals as well as that $b$ ) $\xi(t)$ remains strictly within a compact subset of $\Omega_{\xi}$, which leads by contradiction to $\tau_{\max }=\infty$ and consequently to the completion of the proof.

Phase A. Selecting the parameters $\underline{M}_{p_{i}}, \bar{M}_{p_{i}}, i=$ $1, \ldots, N$ according to (7), we guarantee that the set $\Omega_{\xi}$ is nonempty and open. Moreover, as shown in (8) owing to Assumption A1, $\xi_{p}(0) \in \Omega_{\xi_{p}}$. Furthermore, selecting 
$\rho_{v_{i}}(0)>\left|e_{v_{i}}(0)\right|, i=1, \ldots, N$ guarantees that $\xi_{v}(0) \in$ $\Omega_{\xi_{v}}$ as well. Thus, we conclude that $\xi(0) \in \Omega_{\xi}$. Additionally, $h$ is continuous on $t$ and locally Lipschitz on $\xi$ over the set $\Omega_{\xi}$. Therefore, the hypotheses of Theorem 1 stated in Subsection II-B hold and the existence of a maximal solution $\xi(t)$ of (18) on a time interval $\left[0, \tau_{\max }\right)$ such that $\xi(t) \in \Omega_{\xi}$, $\forall t \in\left[0, \tau_{\max }\right)$ is ensured.

Phase B. We have proven in Phase A that $\xi(t) \in \Omega_{\xi}$, $\forall t \in\left[0, \tau_{\max }\right)$ and more specifically that:

$$
\left.\begin{array}{l}
\xi_{p_{i}}(t)=\frac{e_{p_{i}}(t)}{\rho_{p_{i}}(t)} \in\left(-\underline{M}_{p_{i}}, \bar{M}_{p_{i}}\right) \\
\xi_{v_{i}}(t)=\frac{e_{v_{i}}(t)}{\rho_{v_{i}}(t)} \in(-1,1)
\end{array}\right\}, i=1, \ldots, N
$$

for all $t \in\left[0, \tau_{\max }\right)$, from which we obtain that $e_{p_{i}}(t)$ and $e_{v_{i}}(t)$ are absolutely bounded by $\max \left\{\underline{M}_{p_{i}}, \bar{M}_{p_{i}}\right\} \rho_{p_{i}}(t)$ and $\rho_{v_{i}}(t)$ respectively for $i=1, \ldots, N$. Furthermore, owing to (19), the error vectors $\varepsilon_{p}\left(\xi_{p}\right)$ and $\varepsilon_{v}\left(\xi_{v}\right)$ as given in (12) and (15) are well defined for all $t \in\left[0, \tau_{\max }\right)$. Therefore, consider the positive definite and radially unbounded function $V_{p}=\frac{1}{2} \varepsilon_{p}^{T} P \varepsilon_{p}$ where $P=\left(\operatorname{diag}\left(\tilde{L}^{-1} \underline{\mathbf{1}}\right)\right)^{-1}$ is a diagonal positive definite matrix satisfying $Q=P \tilde{L}+\tilde{L}^{T} P>0$, as dictated by Lemma 1 . Differentiating $V_{p}$ with respect to time, substituting (11), (16) and exploiting: i) the diagonality of $P, r_{p_{\sim}}\left(\xi_{p}\right), \rho_{p}(t)$ matrices, ii) the positive definiteness of $Q=P \tilde{L}+\tilde{L}^{T} P$ as well as iii) the boundedness of $\dot{\rho}_{p}(t)$, $\rho_{v}(t)$ and $\dot{\bar{p}}_{0}(t)$, we get:

$$
\begin{aligned}
\dot{V}_{p} \leq & -k_{p} \lambda_{\min }(Q)\left\|\varepsilon_{p}^{T} r_{p}\left(\xi_{p}\right)\left(\rho_{p}(t)\right)^{-1}\right\|^{2} \\
& +\left\|\varepsilon_{p}^{T} r_{p}\left(\xi_{p}\right)\left(\rho_{p}(t)\right)^{-1}\right\| \bar{F}_{p}
\end{aligned}
$$

where $\bar{F}_{p}$ is a positive constant independent of $\tau_{\max }$, satisfying:

$$
\left\|P\left(\dot{\rho}_{p}(t) \xi_{p}+\tilde{L}\left(\rho_{v}(t) \xi_{v}-\dot{\bar{p}}_{0}(t)\right)\right)\right\| \leq \bar{F}_{p}
$$

for all $(\xi, t) \in \Omega_{\xi} \times \Re_{+}$. Therefore, we conclude that $\dot{V}_{p}$ is negative when $\left\|\varepsilon_{p}^{T} r_{p}\left(\xi_{p}\right)\left(\rho_{p}(t)\right)^{-1}\right\|>\frac{\bar{F}_{p}}{k_{p} \lambda_{\min }(Q)}$ from which, owing to the positive definiteness and diagonality of $r_{p}\left(\xi_{p}\right)\left(\rho_{p}(t)\right)^{-1}$ as well as employing (6) and (11), it can be easily verified that:

$$
\left\|\varepsilon_{p}(t)\right\| \leq \bar{\varepsilon}_{p}:=\max \left\{\left\|\varepsilon_{p}(0)\right\|, \frac{\bar{F}_{p} \max \left\{\frac{\underline{\underline{M}} p_{i} \bar{M}_{p_{i}}}{\underline{\underline{M} p_{i}+\bar{M}_{p_{i}}}}\right\}}{k_{p} \lambda_{\min }(Q) \lambda_{\min }(P)}\right\}
$$

for all $t \in\left[0, \tau_{\max }\right)$. Furthermore, from (12), taking the inverse logarithmic function, we obtain:

$-\underline{M}_{p_{i}}<\frac{e^{-\bar{\varepsilon} p_{p}}-1}{e^{\bar{\varepsilon}_{p}}+1} \underline{M}_{p_{i}}=\underline{\xi}_{p_{i}} \leq \xi_{p_{i}}(t) \leq \bar{\xi}_{p_{i}}=\frac{e^{\frac{\bar{\varepsilon}_{p}}{\varepsilon^{\prime}}-1}}{e^{\bar{\varepsilon}_{p}}+1} \bar{M}_{p_{i}}<\bar{M}_{p_{i}}$

for all $t \in\left[0, \tau_{\max }\right)$ and $i=1, \ldots, N$. Thus, the reference velocity vector $v_{d}\left(\xi_{p}, t\right)$, as designed in (10), remains bounded for all $t \in\left[0, \tau_{\max }\right)$. Moreover, invoking $v_{i}=v_{d_{i}}+\rho_{v_{i}}(t) \xi_{v_{i}}, i=1, \ldots, N$ we also conclude the boundedness of the velocities $v_{i}(t), i=1, \ldots, N$ for all $t \in\left[0, \tau_{\max }\right)$. Finally, differentiating $v_{d}\left(\xi_{p}, t\right)$ with respect to time, substituting (16) and utilizing (22), it is straightforward to deduce the boundedness of $\dot{v}_{d}$ for all $t \in\left[0, \tau_{\max }\right)$ as well.

Applying the aforementioned line of proof, considering the positive definite and radially unbounded function $V_{v}=$ $\frac{1}{2} \varepsilon_{v}^{T} M \varepsilon_{v}$ where $M=\operatorname{diag}\left(\left[m_{i}\right]_{i=1, \ldots, N}\right)$ with $m_{i}, i=$ $1, \ldots, N$ denoting the unknown mass of the vehicle model (1), we conclude that:

$$
\left\|\varepsilon_{v}(t)\right\| \leq \bar{\varepsilon}_{v}:=\max \left\{\left\|\varepsilon_{v}(0)\right\|, \frac{\bar{F}_{v}}{2 k_{v} \min \left\{m_{i}\right\}}\right\}
$$

for all $t \in\left[0, \tau_{\max }\right)$, where $\bar{F}_{v}$ is a positive constant satisfying:

$$
\left\|\left(M \dot{\rho}_{v}(t) \xi_{v}-\left(f\left(v_{d}+\rho_{v}(t) \xi_{v}\right)+w(t)\right)+\dot{v}_{d}\right)\right\| \leq \bar{F}_{v}
$$

owing to: i) the boundedness of $v_{d}$ and $\dot{v}_{d}$ that was proven previously, ii) the continuity of function $f(\cdot)$ and iii) the boundedness of $\dot{\rho}_{v}(t), \rho_{v}(t)$ as well as of the disturbance term $w(t)$. Furthermore, from (15), taking the inverse logarithmic function, we obtain:

$$
-1<\frac{e^{-\bar{\varepsilon}_{v}}-1}{e^{-\bar{\varepsilon}_{v}+1}}=\underline{\xi}_{v_{i}} \leq \xi_{v_{i}}(t) \leq \bar{\xi}_{v_{i}}=\frac{e^{\bar{\varepsilon}_{v}}-1}{e^{\bar{\varepsilon}_{v}}+1}<1
$$

for all $t \in\left[0, \tau_{\max }\right)$ and $i=1, \ldots, N$, which also leads to the boundedness of the distributed control protocol (14).

Up to this point, what remains to be shown is that $\tau_{\max }$ can be extended to $\infty$. In this direction, notice by (22) and (25) that $\xi(t) \in \Omega_{\xi}^{\prime}=\Omega_{\xi_{p}}^{\prime} \times \Omega_{\xi_{v}}^{\prime}, \forall t \in\left[0, \tau_{\max }\right)$, where:

$$
\begin{aligned}
& \Omega_{\xi_{p}}^{\prime}=\left[\underline{\xi}_{p_{1}}, \bar{\xi}_{p_{1}}\right] \times \cdots \times\left[\underline{\xi}_{p_{N}}, \bar{\xi}_{p_{N}}\right] \\
& \Omega_{\xi_{v}}^{\prime}=\left[\underline{\xi}_{v_{1}}, \bar{\xi}_{v_{1}}\right] \times \cdots \times\left[\underline{\xi}_{v_{N}}, \bar{\xi}_{v_{N}}\right]
\end{aligned}
$$

are nonempty and compact subsets of $\Omega_{\xi_{p}}$ and $\Omega_{\xi_{v}}$ respectively. Hence, assuming $\tau_{\max }<\infty$ and since $\Omega_{\xi}^{\prime} \subset \Omega_{\xi}$, Proposition 1 in Subsection II-B dictates the existence of a time instant $t^{\prime} \in\left[0, \tau_{\max }\right)$ such that $\xi\left(t^{\prime}\right) \notin \Omega_{\xi}^{\prime}$, which is a clear contradiction. Therefore, $\tau_{\max }=\infty$. Thus, all closed loop signals remain bounded and moreover $\xi(t) \in \Omega_{\xi}^{\prime} \subset$ $\Omega_{\xi}, \forall t \geq 0$. Finally, multiplying (22) by $\rho_{p_{i}}(t)$, we also conclude:

$$
-\underline{M}_{p_{i}} \rho_{p_{i}}(t)<\underline{\xi}_{p_{i}} \rho_{p_{i}}(t) \leq e_{p_{i}}(t) \leq \bar{\xi}_{p_{i}} \rho_{p_{i}}(t)<\bar{M}_{p_{i}} \rho_{p_{i}}(t)
$$

for all $i=1, \ldots, N$ as well as $t \geq 0$ and consequently the solution of the robust formation control problem with prescribed performance under collision and connectivity constraints for the considered platoon of vehicles.

Remark 2: From the aforementioned proof it can be deduced that the proposed control scheme achieves its goals without resorting to the need of rendering $\bar{\varepsilon}_{p}, \bar{\varepsilon}_{v}$ arbitrarily small by adopting extreme values of the control gains $k_{p}$ and $k_{v}$ (see (21) and (23)). More specifically, notice that (22) and (25) hold no matter how large the finite bounds $\bar{\varepsilon}_{p}, \bar{\varepsilon}_{v}$ are. In the same spirit, large model uncertainties can be compensated, as they affect only the size of $\bar{\varepsilon}_{v}$ 
through $\bar{F}_{v}$ (see (24)), but leave unaltered the achieved stability properties. Hence, the actual performance given in (26), which is solely determined by the functions $\rho_{p_{i}}(t)$ and the parameters $\underline{M}_{p_{i}}, \bar{M}_{p_{i}}, i=1, \ldots, N$, becomes isolated against model uncertainties, thus extending greatly the robustness of the proposed control scheme. Furthermore, unlike what is the common practice in the related literature (i.e., the control gains are tuned towards satisfying a desired performance, nonetheless without any a priori guarantees), the selection of the control gains $k_{p}$ and $k_{v}$ is significantly simplified to adopting those values that lead to reasonable control effort.

\section{Simulation Results}

To demonstrate the efficiency of the proposed distributed control protocol, we consider a platoon of $N=10$ vehicles with the following nonlinear model:

$$
\begin{aligned}
\dot{p}_{i} & =v_{i} \\
1.2 \dot{v}_{i} & =-0.5 v_{i}-0.25\left|v_{i}\right| v_{i}+u_{i}+A_{i} \sin \left(\omega_{i} t+\phi_{i}\right)
\end{aligned}
$$

with $A_{i}, \omega_{i}, \phi_{i}$ randomly chosen in $[1.0,1.5],[2.0,2.5]$ and $[0,2 \pi]$ respectively. The leader node follows a constant velocity model given by $p_{0}(t)=1.5 t$. Furthermore, the desired distance between consecutive vehicles is equally set at $\Delta_{i-1, i}=\Delta^{\star}=0.75, i=1, \ldots, 10$ whereas the collision and connectivity constraints are given by $\Delta_{\text {col }}=0.05 \Delta^{\star}$ and $\Delta_{\text {con }}=1.95 \Delta^{\star}$ respectively. Notice that the aforementioned formation problem under the collision/connectivity constraints is feasible since $\Delta_{\text {col }}<\Delta_{i-1, i}<\Delta_{\text {con }}, i=$ $1, \ldots, 10$. Moreover, we also require steady state errors of no more than $0.05 \mathrm{~m}$ and minimum speed of convergence as obtained by the exponential $e^{-0.5 t}$. Thus, according to (7) we selected the parameters $\underline{M}_{p_{i}}=\bar{M}_{p_{i}}=0.95 \Delta^{\star}, i=$ $1, \ldots, 10$ and the functions $\rho_{p_{i}}(t)=\left(1-\frac{0.05}{0.95 \Delta^{\star}}\right) e^{-0.5 t}+$ $\frac{0.05}{0.95 \Delta^{\star}}, i=1, \ldots, 10$ in order to achieve the desired transient and steady state performance specifications as well as to comply with the collision and connectivity constraints. Furthermore, we chose $\rho_{v_{i}}(t)=2\left|e_{v_{i}}(0)\right| e^{-0.5 t}+0.1$, $i=1, \ldots, 10$ and $k_{p}=k_{v}=0.25$ to yield reasonable control effort. More specifically, the distance between subsequent vehicles along with the collision and connectivity constraints are pictured in Fig. 2. As it was predicted by the theoretical analysis, the formation control problem with prescribed transient and steady state performance is solved with bounded closed loop signals, despite the presence of external disturbances as well as the lack of knowledge of the vehicles' dynamics. Finally, a short video of the simulation results can be found in: http://youtu.be/1zhEau0SIpQ.

\section{REFERENCES}

[1] W. S. Levine and M. Athans, "On the optimal error regulation of a string of moving vehicles," IEEE Transactions on Automatic Control, vol. 11, pp. 355-361, 1966.

[2] S. M. Melzer and B. C. Kuo, "Optimal regulation of systems described by a countably infinite number of objects," Automatica, vol. 7, no. 3, pp. 359-366, 1971.

[3] S. E. Shladover, "Longitudinal control of automotive vehicles in closeformation platoons," Journal of Dynamic Systems, Measurement, and Control, vol. 113, no. 2, pp. 231-241, 1991.

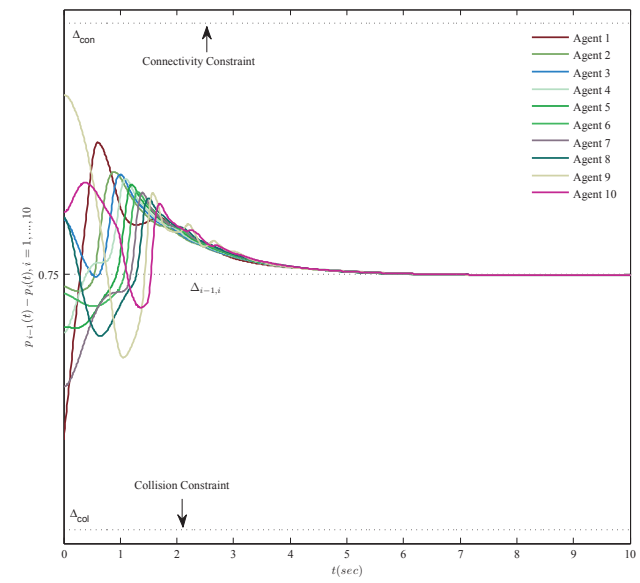

Fig. 2. The distance between successive vehicles along with the collision and connectivity constraints.

[4] M. R. Jovanovic and B. Bamieh, "On the ill-posedness of certain vehicular platoon control problems," IEEE Transactions on Automatic Control, vol. 50, no. 9, pp. 1307-1321, 2005.

[5] S. S. Stankovic, M. J. Stanojevic, and D. D. Siljak, "Decentralized overlapping control of a platoon of vehicles," IEEE Transactions on Control Systems Technology, vol. 8, no. 5, pp. 816-832, 2000.

[6] M. E. Khatir and E. J. Davison, "Decentralized control of a large platoon of vehicles using non-identical controllers," in Proceedings of the American Control Conference, vol. 3, 2004, pp. 2769-2776.

[7] H. Hao and P. Barooah, "Stability and robustness of large platoons of vehicles with double-integrator models and nearest neighbor interaction," International Journal of Robust and Nonlinear Control, vol. 23, no. $18,2013$.

[8] L. E. Peppard, "String stability of relative-motion pid vehicle control systems." IEEE Transactions on Automatic Control, vol. AC-19, no. 5, pp. 579-581, 1974.

[9] Y. Zhang, E. B. Kosmatopoulos, P. A. Ioannou, and C. C. Chien, "Autonomous intelligent cruise control using front and back information for tight vehicle following maneuvers," IEEE Transactions on Vehicular Technology, vol. 48, no. 1, pp. 319-328, 1999.

[10] P. Seiler, A. Pant, and K. Hedrick, "Disturbance propagation in vehicle strings," IEEE Transactions on Automatic Control, vol. 49, no. 10, pp. 1835-1841, 2004.

[11] P. Barooah, P. G. Mehta, and J. P. Hespanha, "Mistuning-based control design to improve closed-loop stability margin of vehicular platoons," IEEE Transactions on Automatic Control, vol. 54, no. 9, pp. 21002113, 2009.

[12] S. El-Ferik, A. Qureshi, and F. L. Lewis, "Neuro-adaptive cooperative tracking control of unknown higher-order affine nonlinear systems," Automatica, vol. 50, no. 3, pp. 798-808, 2014.

[13] Z. Qu, Cooperative Control of Dynamical Systems: Applications to Autonomous Vehicles. New York, USA: Springer, 2009.

[14] A. Berman and R. J. Plemmons, Nonnegative Matrices in the Mathematical Science, ser. Classics in Applied Mathematics. Philadelphia, USA: SIAM, 1994.

[15] C. P. Bechlioulis and G. A. Rovithakis, "Robust adaptive control of feedback linearizable mimo nonlinear systems with prescribed performance," IEEE Transactions on Automatic Control, vol. 53, no. 9, pp. 2090-2099, 2008.

[16] - "Adaptive control with guaranteed transient and steady state tracking error bounds for strict feedback systems," Automatica, vol. 45, no. 2, pp. 532-538, 2009.

[17] — $—$ "Prescribed performance adaptive control for multi-input multioutput affine in the control nonlinear systems," IEEE Transactions on Automatic Control, vol. 55, no. 5, pp. 1220-1226, 2010.

[18] E. D. Sontag, Mathematical Control Theory. London, U.K.: Springer, 1998. 